\title{
Stochastic modeling of the number of felled trees in selection stands
}

\author{
SLOBODANKA S. MITROVICH \\ Faculty of Forestry, Belgrade, Serbia \\ E-mail: minatas@eunet.yu
}

\begin{abstract}
This paper solves the problem of forecasting the number of trees in the selection stands predicted for harvesting in a future period, so that the main resource of the number of trees is sustained. This is achieved by stochastic modeling of the number of felled trees and by solving the certain partial differential equation.
\end{abstract}

Mathematical subject classification: $60 \mathrm{H} 15,35 \mathrm{Q} 80,91 \mathrm{~B} 76$.

Key words: random walk, Itô's lemma, lognormal distribution, Itô’s stochastic differential equation, partial differential equation.

\section{Introduction}

The number of trees in selection stands naturally increases in time. If the conditions were ideal, if there were no natural or artificial (due to an anthropogenic impact) removals of trees, the increase would be exponential in the time from $t_{0}$ to $t$, i.e. the dependence would be:

$$
x(t)=x_{0} \cdot e^{\mu\left(t-t_{0}\right)}
$$

where $x(t)$ is the number of trees in the selection stand per hectare depending on time $t ; \mu$ is the factor of constant growth, $x_{0}$ is the initial number of trees in the stand at the moment $t_{0}$.

Let $S(x, t)$ be the number of felled trees, i.e. the planned impact of the anthropogenic factor on the number of trees $x$ in the selection stand. There is a problem 
how to organise and limit the number of trees for felling in a time period $t=T$, without disturbing the resources. In other words, the question is what capacity of the number of trees in the selection stand can be counted on and planned for harvesting at a definite future moment, so as to maintain the natural process of growth. The significance of the problem is manifold: environmental and economic, bearing in mind that taking care of environmental conditions results in long-term economic gains.

In this paper, the dependencies $x(t)$ and $S(x, t)$ are modeled by Itô's stochastic differential equations and the distribution for the random process $x(t)$ is given. Then the decision on the allowable cut is based on the requirement that the ratio of the changed number of trees in the selection stand and the changed number of felled trees is balanced. This is achieved by solving the derived partial differential equation of the second order, with two boundary conditions and one final condition.

\section{Modeling the number of trees in the stand}

If we observe a relative change $\frac{d x(t)}{x(t)}$ of $x(t)>0$ in a short time interval, we may conclude that it is broken down into the predictable, deterministic part and the random part, with white noise in its base. This is the standard way to explain many natural processes of growth. In other words, the continuous random process $x(t)$ can be represented by Itô's stochastic differential equation:

$$
d x=\mu x d t+\sigma x d w,
$$

where $\mu$ is the drift, the expected rate of return, $\sigma$ is the volatility, the measure of standard deviation, and $w$ is the standard Wiener process. The first term in the above relation is deterministic and the second one is characterised by the presence of the Wiener process as the carrier of randomness. If $\sigma=0$ (there were no removals of trees in selection stand), from the differential equation $d x=\mu x d t$, we can easily get the solution (1) for the process $x$.

Assume that $\mu$ and $\sigma$ are constants in the definite time period. The parameters $\mu$ and $\sigma$ are given in time units, mainly for a year. Often, $\mu$ is not changeable for one kind of selection stand. In the spatial model they are taken to be constant 
values, previously determined by statistical methods based on field data (look at statistical data from [5]).

According to (2), it easy to see that $\ln \frac{x}{x_{0}}$ has a normal distribution with:

$$
E\left(\ln \frac{x}{x_{0}}\right)=\left(\mu-\frac{1}{2} \sigma^{2}\right) t, \quad D\left(\ln \frac{x}{x_{0}}\right)=\sigma^{2} t,
$$

where $t_{0}=0, w\left(t_{0}\right)=0, x(0)=x_{0}$. It means that $\frac{x}{x_{0}}$ has a lognormal distribution with density:

$$
\frac{x_{0}}{\sigma x \sqrt{2 \pi t}} \exp \left(-\frac{\left(\ln \frac{x}{x_{0}}-\left(\mu-\frac{1}{2} \sigma^{2}\right) t\right)^{2}}{2 \sigma^{2} t}\right), \quad x>0 .
$$

\section{Requirement derivatives}

Let us introduce $\frac{1}{t} \int_{0}^{t} x(p) d p$ as the continuously sampled arithmetic average of the number of trees. If we denote $I=\int_{0}^{t} x(p) d p$, it is easy to see that $d I=x d t$.

Now the value of the number of felled trees $S(x, t, I)$ depends on three variables: $x, I$ and $t$. Let us suppose that the dependence is expressed by a continuous function with continuous partial derivations

$$
\frac{\partial S}{\partial t}, \frac{\partial S}{\partial x}, \frac{\partial S}{\partial I} \text { and } \quad \frac{\partial^{2} S}{\partial x^{2}} .
$$

Then by applying Itô's lemma:

$$
d S=\frac{\partial S}{\partial t} d t+\frac{\partial S}{\partial x} d x+\frac{\partial S}{\partial I} d I+\frac{1}{2} \sigma^{2} x^{2} \frac{\partial^{2} S}{\partial x^{2}} d t .
$$

By this we get the stochastic differential equation of random walk which represents the process of the number of felled trees.

Let us consider the relation $S(x, t, I)$ and $x(t)$, by introducing a new function, the requirement $\Pi$ :

$$
\Pi=S-\Omega \cdot x,
$$

where $\Omega=\frac{\partial S}{\partial x}$ is chosen (rate of change $S(x, t, I)$ compared to $x(t)$ ). Such an interpretation of $\Omega$ in this model, aims at neutralising the stochastic component in the following step. It is naturally to consider $\Pi$ as the difference between 
$x(t)$ and $S$. In a reality we always consider how many trees we have now in selection stand and how many trees are allowed to fell. So, this difference is in the structural form of the selection management and its value shouldn't be interrupted.

From the natural assumption that in a short time interval $d t$, the number of trees in the selection stand is not disturbed and that the ratio of the number of trees and the number of felled trees is constant, we get:

$$
\begin{aligned}
d \Pi & =d S-\Omega \cdot d x=d S-\frac{\partial S}{\partial x} d x= \\
& =\frac{\partial S}{\partial t} d t+\frac{\partial S}{\partial x} d x+x \frac{\partial S}{\partial I} d t+\frac{1}{2} \sigma^{2} x^{2} \frac{\partial^{2} S}{\partial x^{2}} d t-\frac{\partial S}{\partial x} d x= \\
& =\left(\frac{\partial S}{\partial t}+x \frac{\partial S}{\partial I}+\frac{1}{2} \sigma^{2} x^{2} \frac{\partial^{2} S}{\partial x^{2}}\right) d t=0
\end{aligned}
$$

Therefore:

$$
\frac{\partial S}{\partial t}+x \frac{\partial S}{\partial I}+\frac{1}{2} \sigma^{2} x^{2} \frac{\partial^{2} S}{\partial x^{2}}=0
$$

and the determination of $S(x, t, I)$ means the solution of (3), a linear partial differential equation of the second order PDE, parabolic type. There is a problem in solving of this PDE. Typically the value of $S$ must be calculed numerically. If we suppose that $S(x, t, I)$ has the form $x \cdot F(R, t)$, where $R=I / x$, we can reduce the problem from three to two dimension.

According to partial derivations of $S(x, t, I)=x \cdot F(R, t)$ :

$$
\frac{\partial S}{\partial t}=x \cdot \frac{\partial F}{\partial t}, \quad \frac{\partial S}{\partial I}=\frac{\partial F}{\partial R}, \quad \frac{\partial^{2} S}{\partial x^{2}}=R^{2} \cdot \frac{1}{x} \cdot \frac{\partial^{2} F}{\partial R^{2}},
$$

we have the new PDE:

$$
\frac{\partial F}{\partial t}+\frac{\partial F}{\partial R}+\frac{1}{2} \sigma^{2} R^{2} \frac{\partial^{2} F}{\partial R^{2}}=0 .
$$

\section{Boundary conditions and final condition}

First, we are analysing for a $t$, when $R$ tends to 0 , the behaviour of $R$. Since

$$
R(x(t), t)=(1 / x) \int_{0}^{t} x(p) d p,
$$


$R$ satisfies the stochastic differential equation

$$
d R=\frac{\partial R}{\partial t} d t+\frac{\partial R}{\partial x} d x+\frac{1}{2} \sigma^{2} x^{2} \frac{\partial^{2} R}{\partial x^{2}} d t .
$$

According to

$$
d x=x \mu d t+\sigma x d w, \quad \frac{\partial R}{\partial t}=1, \quad \frac{\partial R}{\partial x}=-R / x, \quad \frac{\partial^{2} R}{\partial x^{2}}=2 R / x^{2},
$$

we have

$$
d R=\left(1+\left(\sigma^{2}-\mu\right) R\right) d t-R \sigma d w .
$$

From $R \rightarrow 0$, we get $d R=d t>0$. So we only know that in the case $R \rightarrow 0$, $F$ is finite. If $R$ tends to 0 , in the equation (4):

$$
\frac{\partial F}{\partial t}+\frac{\partial F}{\partial R}+\frac{1}{2} \sigma^{2} R^{2} \frac{\partial^{2} F}{\partial R^{2}}=0
$$

we can ignore $R^{2} \frac{\partial^{2} F}{\partial R^{2}}$.

$$
\text { If: } R^{2} \frac{\partial^{2} F}{\partial R^{2}}=O(1), \text { then } \frac{\partial^{2} F}{\partial R^{2}}=O\left(1 / R^{2}\right), \text { or } F=O(\log R) \text {. }
$$

This is inconsistent with $F$ being finite. So it remains:

$$
\frac{\partial F}{\partial t}+\frac{\partial F}{\partial R}=0, \text { when } R \rightarrow 0 .
$$

On the other hand it is clear that:

$$
x \cdot F(\infty, t)=0 .
$$

Since $x$ is bounded for a $t$, the only way that $R=I / x$, increases is $x$ tends to zero. In this case there will not be felling.

These are boundary conditions.

Finally, we have to determine the final condition. Naturally, the number of felled trees in future time $T$, will be a difference between the number of tree in the stand and the continuously sampled arithmetic average of the number of tree from the beginning time $t=0$ to $T$ :

$$
S(x, T, I)=\max (x(T)-I / T, 0)=x \cdot \max (1-R / T, 0) .
$$

It is clear that for $I / T>x$, there will be no felling. It is naturally expecting that for $\mu>0$, the value of growth $x(t)$ is great than the average value of $x$ from time 0 to $t$. Condition (7) is the final condition for PDE (3). 


\section{Solving PDE}

In order to solve PDE (4) we seek a solution of the form:

$$
F(R, t)=p(t)+q(t) \cdot R
$$

where $R=I / x$, and from the final condition: $p(T)=1, q(T)=-1 / T$. According to partial derivations:

$$
\frac{\partial F}{\partial t}=p^{\prime}(t)+q^{\prime}(t) \cdot R, \quad \frac{\partial F}{\partial R}=q(t), \quad \frac{\partial^{2} F}{\partial R^{2}}=0,
$$

and the condition (5) we obtain: $p^{\prime}(t)=-q(t)$, and $q^{\prime}(t)=0$. Finally we have: $p(t)=t / T, q(t)=-1 / T$,

$$
F(R, t)=\frac{t}{T}-\frac{R}{T}, \quad S(x, t, I)=x \cdot F(R, t)=\frac{t \cdot x(t)}{T}-\frac{1}{T} \int_{0}^{t} x(p) d p .
$$

And the explicit expression for $S(x, t, I)$ in time $T$ is:

$$
S(x, T, I)=x(T)-\frac{1}{T} \int_{0}^{T} x(p) d p,
$$

which represents the maximal number of wood allowed to cut in time $T$.

\section{Examples}

Based on statistical data measured in the Management Unit "Tara" - National Park "Tara", Bajina Basta, Serbia, between 1960 and 1990, several times the number of felled trees amounted up to even $20 \%$ of the number of trees in the stand. Also, by comparing only the numbers of trees in the stand in a ten-year period, it was calculated that these values were statistically equal (see [5]), which leads to a conclusion that the method of selection forests management applied in the forests of Tara in the past decades was relatively successful. In other words, the initial resource of the number of trees was maintained, and the quantity selected for felling was only the percentage that accumulated during ten years, like an "interest". 
Example. In 1970, the number of trees in the selection stand, Compartment 51, Management Unit "Tara", was $x(t)=557.4$, (with the expected rate of return $\mu=0.02$, and the standard deviation $\sigma=0.2$ ). On the same sample plot in 1980 the number of trees was 680.4 , (see [5]). By the data from the ten years period we can calculate $I / 10=\frac{1}{10} \int_{0}^{10} x(p) d p=579.3$. Now, we get $S(x, t+10)$ as an allowed number of trees selected for felling $S=101.1$ in 1980 .

In 1980 the number of felled trees was 109.1 per hectare (see [5]), which is little greater than the maximal number of trees selected for felling, calculated by theoretical means.

Example. In 1960, the number of trees in the selection stand, Compartment 62 , Management Unit “Tara”, was $x(t)=664.0$ (with the expected rate of return $\mu=0.02$, and the standard deviation $\sigma=0.2$ ). On the same sample plot in 1970 the number of trees was 811.0, (see [5]). By the data from the ten years period we can calculate $I / 10=\frac{1}{10} \int_{0}^{10} x(p) d p=693.2$. Now, we get $S(x, t+10)$ as an allowed number of trees selected for felling $S=117.8$ in 1970 .

In 1970 the number of felled trees was 126.4 per hectare (see [5]), which is little greater than the maximal number of trees selected for felling, calculated by theoretical means.

\section{Conclusion}

As the permanent maintenance of selection structure is possible only by systematic selection management, primarily by selection cuttings the above stochastic analysis of the number of trees and the number of the felled trees during a longer time period can point to the success-failure of the performed selection system of management. The primary objective of this type of study is to predict based on a stochastic model effect of the scope of selection cuttings and the number of trees for recruitment. The simulation of growth is useful to foresee the future development of selection stands.

The above examples point out that the field data on the number of felled trees, correspond completely to the theoretical upper boundaries. This means that the calculated forecast for $S(x, t)$ can also be applied in future for the assessment of the upper permissible limit of the number of trees for felling. 


\section{REFERENCES}

[1] K. Itô, On stochastic Differential Equations, Memorial Mathematical Society, (1951), 1-51.

[2] P. Wilmott, S. Howison and J. Dewynne, Mathematics of Financial Derivatives, Cambridge University Press, (1995).

[3] J.M. Steele, Stochastic Calculus and Financial Applications, Spinger-Verlag New York Inc., (2001).

[4] F. Black and M. Scholes, The pricing of options and corporate liabilities, Journal of Political Economy, 81(3) (1973), 637-659.

[5] S. Mitrovich and S. Bankovich, The Possibilities of Using Group Regression in the Analysis of the Diameter Structure Development in Selection Forests of Tara, University in Belgrade, Bulletin of the Faculty of Forestry, 85 (2002), 79-90. (in Serbian with summary in English).

[6] S. Mitrovich, Stochastic modeling of the number of trees and the number of felled trees in selection stands, YUJOR, Belgrade, 14(1), (2004), pp. 57-64. 